\title{
Photonic crystal slab between orthogonal polarizers: details on the guided mode resonance wavelength
}

\author{
Hannes Lüder ${ }^{1}$ (D) $\cdot$ Moritz Paulsen $^{1}$ (D) $\cdot$ Martina Gerken $^{1}$ (D)
}

Received: 30 September 2019 / Accepted: 4 March 2020 / Published online: 13 March 2020

(c) The Author(s) 2020

\begin{abstract}
The transmission spectrum of a photonic crystal slab features sharp dips created by guided mode resonances. The same photonic crystal slab placed between orthogonal polarizers shows peaks at the resonances, but the peak wavelength differs from the guided mode resonance wavelength by a few nanometres. We investigate the working principle of the orthogonal polarizer setup and the origin of the wavelength difference for the case of a TE resonance. We show that the peak in the orthogonal polarizer setup is formed by light from the non-resonant TM polarization. The wavelength difference is caused by the phase shift between the resonant TE and the non-resonant TM polarization. We compare our explanation to a temporal coupled-mode approach and the use of a time-domain window function in FDTD.
\end{abstract}

Keywords Photonic crystal slab · Resonant waveguide grating · Guided mode resonance · Fano resonance $\cdot$ Resonance position $\cdot$ Orthogonal polarization filters · Jones calculus · Temporal coupled-mode theory $\cdot$ FDTD

This article is part of the Topical Collection on Optical Wave and Waveguide Theory and Numerical Modelling.

Guest edited by Alejandro Ortega Moñux, Rafael Godoy Rubio and Jiri Ctyroky.

Hannes Lüder

halu@tf.uni-kiel.de

Moritz Paulsen

mail@moritz-paulsen.de

Martina Gerken

mge@tf.uni-kiel.de

https://www.isp.tf.uni-uiel.de

1 Institute of Electrical Engineering and Information Technology, Kiel University, Kaiserstraße 2, 24143 Kiel, Germany 


\section{Introduction}

A photonic crystal slab (PCS), also known as resonant waveguide grating (RWG), is a planar optical waveguide with an integrated periodic nanostructure (essentially a periodic refractive index modulation). The nanostructure leads to the formation of quasi-guided (Bloch-) modes (also called leaky modes), which couple to the continuum of radiation modes. This coupling gives rise to characteristic narrowband features in the transmission and reflection spectrum when the photonic crystal slab is illuminated from top or bottom. (Johnson et al. 1999; Quaranta et al. 2018).

When light is incident on a photonic crystal slab, it is not only directly transmitted and reflected, but also coupled into the grating waveguide, which acts as an open resonator, and excites one or multiple quasi-guided modes. Because these modes couple to the outside, they create resonance effects leading (for a lossless dielectric structure) to perfect reflection and zero transmission for one or multiple specific wavelengths, which are called 'guided mode resonance wavelengths' $\left(\lambda_{\mathrm{GMR}}\right.$ ) (Wang and Magnusson 1993; Fan and Joannopoulos 2002; Hermannsson et al. 2014). Figure 1 shows measured transmission spectra for a 1D photonic crystal slab. In the figures throughout this paper, blue indicates TE, red indicates $\mathrm{TM}$, and green indicates the orthogonal polarizer transmission.

Because a resonance wavelength's position can be tuned by and is sensitive to changing the structure's period and the materials' refractive indices, photonic crystal slabs are used, e.g., as optical filters and sensors (Pitruzzello and Krauss 2018; Kilic et al. 2008). For compact optical systems and also for characterization measurements in the lab it can be advantageous to place a one-dimensional photonic crystal slab between two orthogonal polarizers (OP), aligned at $45^{\circ}$ and $-45^{\circ}$ to the grating lines, respectively (Nazirizadeh et al. 2008). This setup suppresses the background light and converts the dip in the transmission spectrum into a peak, enabling a simple intensity-based readout instead of a spectrometric

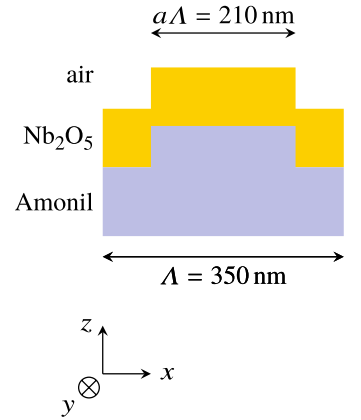

(a)

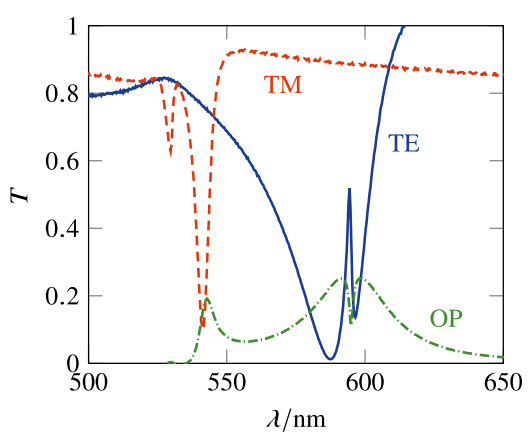

(b)

Fig. 1 a Unit cell of a fabricated one-dimensional photonic crystal slab. The high-index layer is formed by a sputtered $85 \mathrm{~nm}$ niobium pentoxide layer $(n \approx 2.3)$. The grating (period $\Lambda=350 \mathrm{~nm}$, duty cycle $a=0.6$, height $=60 \mathrm{~nm})$ is fabricated by nanoimprint lithography into an Amonil layer $(n \approx 1.5)$. b Transmission measurements for TE and TM polarization and for the orthogonal polarizer setup. See Fig. 2 for the different measurement setups. Each polarization shows one transmission dip, which corresponds to the quasiguided TE and TM mode, respectively. Both transmission dips translate into transmission peaks in the orthogonal polarizer setup. The peak artefacts in the transmission dips are created by the superposition of different angular contributions of the illumination because our measurement setup does not provide a perfectly isolated $0^{\circ}$ measurement 


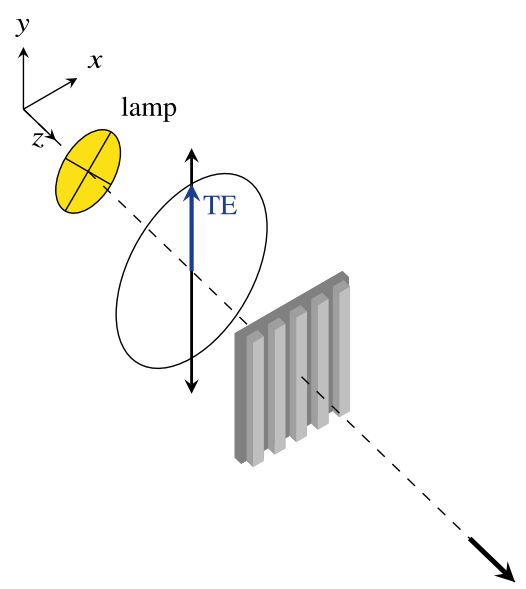

to the

spectrometer

(a)

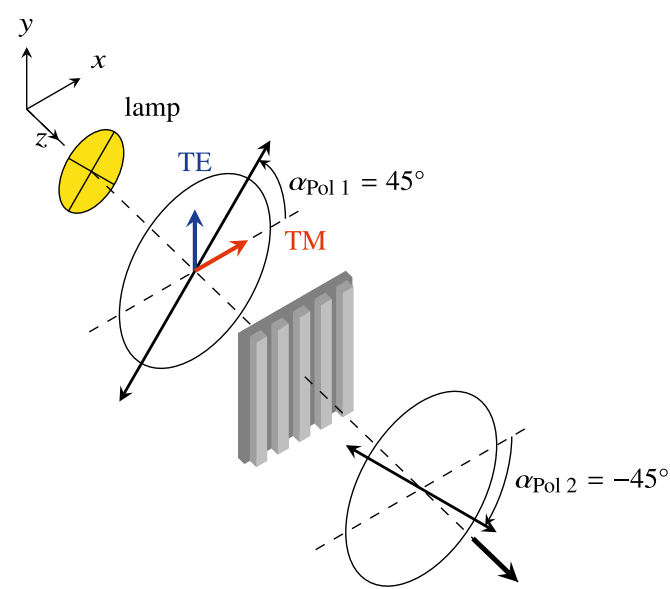

to the

(b)

Fig. 2 Simplified schematics of the polarizer setups under investigation. For the experimental characterization the setup is embedded into a microscope setup that is connected to a spectrometer. a Transmission measurement for TE polarized light. The TM transmission setup is identical, only the polarizer is rotated by $90^{\circ}$. b Orthogonal polarizer setup. The polarizers are perpendicular to each other and oriented at $\pm 45^{\circ}$ to the grating

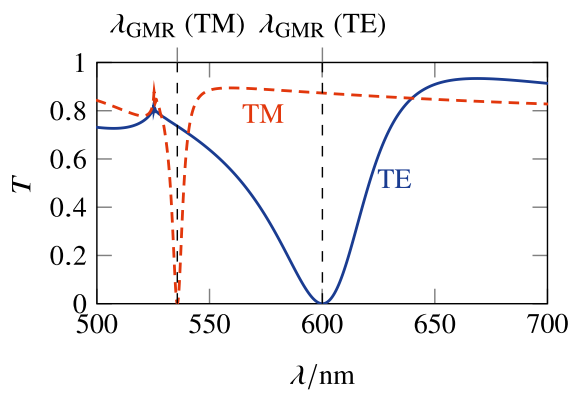

(a)

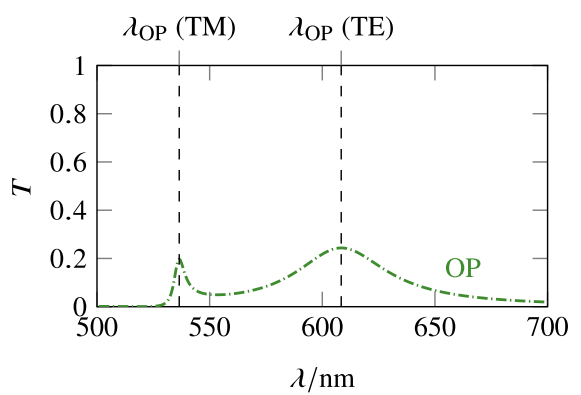

(b)

Fig. 3 Simulated transmission spectra for the photonic crystal slab shown in Fig. 1a. a TE and TM, b orthogonal polarizer setup. The different resonance wavelengths' positions are indicated for the TE and TM resonance: $\lambda_{\mathrm{GMR}}(\mathrm{TM})=535.7 \mathrm{~nm}, \lambda_{\mathrm{GMR}}(\mathrm{TE})=600.1 \mathrm{~nm}, \lambda_{\mathrm{OP}}(\mathrm{TM})=536.5 \mathrm{~nm}, \lambda_{\mathrm{OP}}(\mathrm{TE})=608.4 \mathrm{~nm}$

one (Nazirizadeh et al. 2010; Lin et al. 2014; Jahns et al. 2015). The measurement setups for direct and orthogonal polarizer setup measurements are schematically shown in Fig. 2.

We found both experimentally and theoretically that the wavelength of the resonance maximum with the orthogonal polarizer setup ( $\lambda_{\mathrm{OP}}$, see Fig. 3a) does not exactly agree with the aforementioned $\lambda_{\mathrm{GMR}}$ (see Fig. 3b), but they differ by a few nanometres (depending on the particular structure under investigation). To our knowledge, this effect has not yet been discussed in the literature. The wavelength difference $\Delta \lambda=\left|\lambda_{\mathrm{OP}}-\lambda_{\mathrm{GMR}}\right|$ is important when the precise value of the absolute resonance wavelength is of interest (Hermannsson et al. 2014). 
In this paper, we present a consistent and easy-understandable explanation for the wavelength difference based on the amplitudes and phases of the transmitted TE and TM polarized waves. The effect is also explainable by directly using the concept of Fano resonances, but we experienced that a more concrete explanation is easier to understand especially for students who are newly introduced to the topic of photonic crystal slabs.

Because our aim is to keep the explanations easy to understand, we start with a short introduction to photonic crystal slab fundamentals, where we also introduce the concept of Jones vectors and Jones matrices for a consistent description of polarization states and polarizing systems, and continue with a rather detailed analysis of the orthogonal polarizer setup. After that we deduce the origin of the discrepancy between the observed resonance wavelength for the different polarizer setups. Finally, we compare our explanation to other approaches from the literature.

For the theoretical analysis, we use the finite-difference time-domain method [FDTD, Lumerical FDTD Solutions (Lumerical Inc. 2019)] to calculate transmission spectra and the finite element method [FEM, Comsol Multiphysics, Waveoptics module COMSOL Multiphysics (2019)] to calculate eigenmodes of the photonic crystal slab. For all the simulations, we used the same 1D photonic crystal slab, which is shown in Fig. 1a, with the non-dispersive material properties $n_{\text {substrate }}=1.5$ and $n_{\text {high index }}=2.3$.

\section{Photonic crystal slab and Jones calculus fundamentals}

As already stated, a photonic crystal slab is a high refractive index slab with a periodic refractive index modulation (Joannopoulos et al. 2008). In general, one- and two-dimensional modulations are possible. Here, we restrict our analysis to the $1 \mathrm{D}$ case. In that case, the geometry is uniform in $y$ direction (see Figs. 1 or 2 for the definition of the coordinate system used in this work) and therefore all $y$ derivatives vanish. It is easy to see that the source-free, non-magnetic Maxwell's equations in phasor domain for a harmonic time dependence $\exp (\mathrm{i} \omega t)$

$$
\begin{array}{rlrl}
\operatorname{curl} \vec{H} & =\mathrm{i} \omega \varepsilon(\vec{r}) \vec{E} & \operatorname{div} \varepsilon \vec{E}=0 \\
\operatorname{curl} \vec{E}=-\mathrm{i} \omega \mu_{0} \vec{H} & \operatorname{div} \mu_{0} \vec{H}=0
\end{array}
$$

decouple into two independent sets of equations, one for the field components $\left(E_{y}, H_{x}, H_{z}\right)$, called transverse electric (TE) fields, because the electric field is transverse to the plane of interest, and one for the field components $\left(H_{y}, E_{x}, E_{z}\right)$ called transverse magnetic (TM) fields. The TE and TM solutions are independent (van Bladel 2007).

It is instructive to view the modulated waveguide as a periodically perturbed slab waveguide. For the corresponding unperturbed slab waveguide, the TE and TM modes and their dispersion diagrams can be calculated using e.g. a transfer-matrix method (Kwon 2009). For weak perturbations, the modes of the modulated waveguide have dispersion relations similar to those of the slab waveguide modes. But due to the periodic perturbation, the modes are no longer perfectly guided and orthogonal to incoming light waves. Instead, quasi-guided modes (QGM) are formed, which couple to in- and outgoing waves if the periodic structure's grating vector $\vec{K}=2 \pi / \Lambda \overrightarrow{\mathrm{e}}_{x}$ compensates for the wave vector mismatch between the waves: $\pm K+k_{\text {inc }, x}-k_{\mathrm{QGM}}=0$ (Rosenblatt et al. 1997).

Whenever this happens, a resonant interaction of the incoming light with the quasiguided mode emerges, which drastically changes the transmission and reflection 
characteristics of the photonic crystal slab compared to its corresponding unstructured slab waveguide: at the resonance, typically an asymmetric transmission $\operatorname{dip}(T=0 \%)$ and a transmission peak $(T \rightarrow \max )$ are created on the smooth Fabry-Perot background transmission spectrum (Fan and Joannopoulos 2002).

The phenomena we will describe are based on the polarizing properties of photonic crystal slabs. The polarization of a light wave and its interaction with a polarizing system is conveniently described using Jones vectors and Jones matrices (Hecht 2002, Chap. 8; Saleh and Teich 2019, Chap. 6). For completeness, we give a short definition of the relevant terms: A $z$-propagating plane wave has the general form

$$
\vec{E}(z, t)=\left(\begin{array}{c}
E_{x}(t) \\
E_{y}(t) \\
0
\end{array}\right)=\operatorname{Re}\left\{\left(\begin{array}{c}
\left|\underline{E}_{x}\right| \exp \left(\mathrm{i} \varphi_{x}\right) \\
\left|\underline{E}_{y}\right| \exp \left(\mathrm{i} \varphi_{y}\right) \\
0
\end{array}\right) \exp (\mathrm{i}(k z-\omega t))\right\}=: \operatorname{Re}\left\{\left(\begin{array}{c}
J_{1} \\
J_{2} \\
0
\end{array}\right) \exp (\mathrm{i}(k z-\omega t))\right\},
$$

where $\underline{E}_{x}=\left|\underline{E}_{x}\right| \exp \left(\mathrm{i} \varphi_{x}\right)$ and $\underline{E}_{y}=\left|\underline{E}_{y}\right| \exp \left(\mathrm{i} \varphi_{y}\right)$ are complex phasors. The Jones vector $\vec{J}$ for this plane wave is defined as $\vec{J}=\left(\underline{E}_{x}, \underline{E}_{y}\right)^{\top}=\left(J_{1}, J_{2}\right)^{\top} \in \mathbb{C}^{2}$. It characterizes the polarisation of the plane wave completely. For example, $\varphi_{x}=\varphi_{y}$ describes linearly polarized waves and $\left|\underline{E}_{x}\right|=\left|\underline{E}_{y}\right|, \varphi_{y}=\varphi_{x} \pm \pi / 2$ circularly polarized waves. In the following, we will normalize the Jones vector of the light wave incident to the photonic crystal slab to $\left|\vec{J}_{\text {in }}\right|^{2}=1$ and $J_{\text {in } 1} \in \mathbb{R}$ at the beginning of a calculation. According to the coordinate system defined in Fig. 2, TE polarized light is described by $\vec{J}_{\mathrm{TE}}=(0,1)^{\mathrm{T}}$, TM polarized light by $\vec{J}_{\mathrm{TM}}=(1,0)^{\mathrm{T}}$ and the normalized equal superposition of both by $\vec{J}_{\mathrm{TETM}}=1 / \sqrt{2}(1,1)^{\mathrm{T}}$.

The change in polarization through a polarizing system is described by a Jones matrix $\mathcal{T} \in \mathbb{C}^{2 \times 2}: \vec{J}_{\text {out }}=\mathcal{T} \cdot \vec{J}_{\text {in }}$. A sequence of systems is described by matrix multiplication: $\vec{J}_{\text {out }}=\mathcal{T}_{n} \cdot \ldots \cdot \mathcal{T}_{2} \cdot \mathcal{T}_{1} \cdot \vec{J}_{\text {in }}$. A linear polarizer, oriented at an angle $\alpha$ to the $x$ axis, has the Jones matrix (Saleh and Teich 2019)

$$
\mathcal{T}_{\alpha}=\left(\begin{array}{cc}
\cos ^{2} \alpha & \cos \alpha \sin \alpha \\
\sin \alpha \cos \alpha & \sin ^{2} \alpha
\end{array}\right) .
$$

\section{Explaining the orthogonal polarizer setup's transmission spectrum}

In this paper, we discuss the setup where a $1 \mathrm{D}$ photonic crystal slab is placed between two orthogonal polarizers, polarized at $45^{\circ}$ and $-45^{\circ}$ to the grating lines, respectively. In this setup, transmission dips of a photonic crystal slab are converted into transmission peaks (Nazirizadeh et al. 2008, 2010). This fact is loosely explained by saying that the orthogonal polarizers suppress all background light, while only the resonant light that interacts with the grating experiences a polarization rotation and can pass the second polarizer.

However, when looking at the resonant case and the electromagnetic fields in detail, one finds that it is actually the non-resonant polarization that passes the second polarizer. Therefore, we will rigorously and quantitatively correctly explain from first principles how the transmission peak is created.

The two polarizers, which are oriented at $\pm 45^{\circ}$ to the $x$ axis, and the photonic crystal slab are described by (Kilic et al. 2008) 


$$
\mathcal{T}_{\mathrm{Pol} 1}=\frac{1}{2}\left(\begin{array}{ll}
1 & 1 \\
1 & 1
\end{array}\right), \quad \mathcal{T}_{\mathrm{Pol} 2}=\frac{1}{2}\left(\begin{array}{ll}
+1 & -1 \\
-1 & +1
\end{array}\right), \quad \text { and } \quad \mathcal{T}_{\mathrm{PCS}}(\lambda)=\left(\begin{array}{cc}
t_{\mathrm{TM}}(\lambda) & 0 \\
0 & t_{\mathrm{TE}}(\lambda)
\end{array}\right),
$$

where $t_{\mathrm{TM}}$ and $t_{\mathrm{TE}}$ describe the wavelength-depended transmission characteristics of the photonic crystal slab. The first polarizer polarizes the incoming unpolarized light to $\vec{J}_{\text {in }}=\vec{J}_{\text {TETM }}=1 / \sqrt{2}(1,1)^{\top}$, which is the starting point for the following analysis. Relative to the grating, which is aligned to the $y$ axis of the coordinate system, the light is linearly polarized at $45^{\circ}$. This polarization state is a superposition of both TE ( $E_{y}$ and $H_{x}$ fields) and TM $\left(H_{y}\right.$ and $E_{x}$ fields) light of equal intensities.

The Jones vectors $\vec{J}_{\text {in }}, \vec{J}_{\text {PCS }}$, and $\vec{J}_{\text {out }}$ describe the polarization after the first polarizer, the photonic crystal slab, and the second polarizer, respectively. The reference points for these vectors and the three Jones matrices are given in Fig. $4 \mathrm{a}$.

Now we distinguish two cases:

- For wavelengths far away from any resonance wavelength, both polarizations pass the photonic crystal slab in a nearly identical way, such that they reach the second polarizer. Because the second polarizer is orthogonally oriented to the first, it blocks the light and the overall transmission is (nearly) zero:

$$
\mathcal{T}_{\mathrm{PCS}} \approx\left(\begin{array}{ll}
1 & 0 \\
0 & 1
\end{array}\right) \Rightarrow \vec{J}_{\text {out }}=\mathcal{T}_{\mathrm{Pol} 2} \cdot \mathcal{T}_{\mathrm{PCS}} \cdot \vec{J}_{\text {in }} \approx\left(\begin{array}{ll}
0 & 0 \\
0 & 0
\end{array}\right) \cdot \vec{J}_{\text {in }}=\left(\begin{array}{l}
0 \\
0
\end{array}\right)
$$

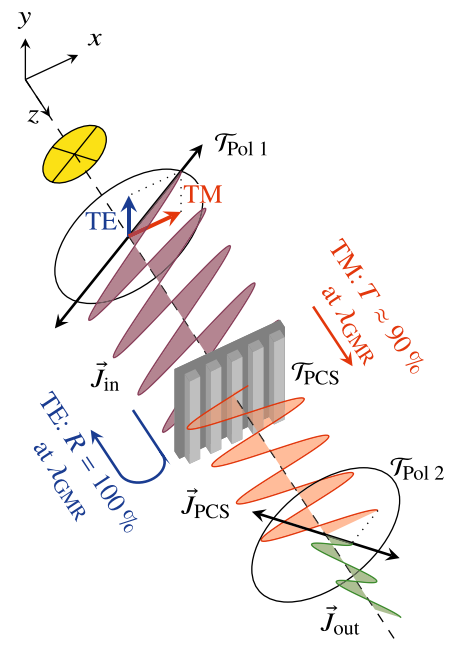

(a)

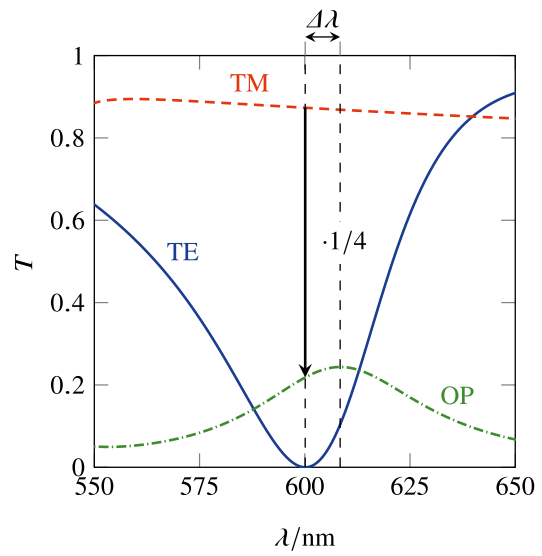

(b)

Fig. 4 a Illustration of the presented explanation for the transmission maximum with orthogonal polarizers. The situation at the TE resonance is depicted. The incident light is linearly polarized by the top polarizer (TE and TM polarization). At $\lambda_{\mathrm{GMR}}$, the TE component is fully reflected (reflected wave not shown). Therefore, only the non-resonant TM light is transmitted and reaches the bottom polarizer, which it can partly pass. The figure also shows the reference points for the three Jones vectors and Jones matrices used in the text. b Detailed view of the transmission spectra at TE resonance from Fig. 3. At the $\lambda_{\mathrm{GMR}}(\mathrm{TE})$, the orthogonal polarizer transmission is exactly $1 / 4$ of the TM transmission. The wavelength difference $\Delta \lambda=\left|\lambda_{\mathrm{OP}}-\lambda_{\mathrm{GMR}}\right|$ is $8.3 \mathrm{~nm}$ 
- At a guided mode resonance, here for example at the TE resonance $\lambda_{\mathrm{GMR}}$ (TE), the TE polarized fraction of the light experiences $100 \%$ reflection at the photonic crystal slab, while the TM polarized light is not resonant at this wavelength and passes the slab basically unchanged. This situation is shown in Fig. 4a. Now, only the TM light (which is non-resonant) reaches the second polarizer, which is oriented $45^{\circ}$ to the $\mathrm{TM}$ light. Therefore, half of the TM light passes the second polarizer and creates a peak in the transmission spectrum:

$$
\mathcal{T}_{\mathrm{PCS}} \approx\left(\begin{array}{ll}
1 & 0 \\
0 & 0
\end{array}\right) \Rightarrow \vec{J}_{\mathrm{out}}=\mathcal{T}_{\mathrm{Pol} 2} \cdot \mathcal{T}_{\mathrm{PCS}} \cdot \vec{J}_{\mathrm{in}} \approx \frac{1}{2} \frac{1}{\sqrt{2}}\left(\begin{array}{c}
+1 \\
-1
\end{array}\right)
$$

The absolute values of the transmitted intensities provide a way to verify this explanation numerically: In Fig. $4 \mathrm{~b}$ we see that the transmission at $\lambda_{\mathrm{GMR}}(\mathrm{TE})$ is only $1 / 4$ of the transmission for the TM case, where just the top polarizer is used to create TM polarized light. This is in agreement with the above calculation, because the reduction of the Jones vector modulus by $1 / 2$ corresponds to an intensity reduction to $1 / 2^{2}=1 / 4$ : The photonic crystal slab reduces the intensity by $1 / 2$, because it transmits only TM light at the TE resonance, and the second polarizer reduces the intensity again by $1 / 2$, because it is at $45^{\circ}$ to the $\mathrm{TM}$ polarization. The observed factor of $1 / 4$ is only possible if the light arriving at the bottom polarizer is indeed purely TM polarized. This proves that at $\lambda_{\mathrm{GMR}}$ (TE) only the non-resonant TM polarization passes the second polarizer.

In this model, one expects the maximum of this peak to be aligned with the guided mode resonance wavelength. Slightly off the resonance wavelength, the nearly-resonant polarization is partly transmitted (e.g. $t_{\mathrm{TM}} \approx 1$ and $t_{\mathrm{TE}} \approx 0.1$ slightly off the TE resonance) and together with the transmitted non-resonant polarization, the overall polarization state of the light after the photonic crystal slab should rotate back to the polarization of the first polarizer. Therefore the overall transmission after the second polarizer should decrease compared to the fully resonant case: for example

$$
\mathcal{T}_{\mathrm{PCS}} \approx\left(\begin{array}{cc}
1 & 0 \\
0 & 0.1
\end{array}\right) \Rightarrow \vec{J}_{\mathrm{out}}=\mathcal{T}_{\mathrm{Pol} 2} \cdot \mathcal{T}_{\mathrm{PCS}} \cdot \vec{J}_{\text {in }} \approx \frac{1}{2} \frac{1}{\sqrt{2}}\left(\begin{array}{c}
+1 \\
-0.9
\end{array}\right) \Rightarrow\left|\vec{J}_{\text {out }}\right|^{2} \approx \frac{0.95}{4}<\frac{1}{4}
$$

\section{Phase and polarization analysis}

However, we observed in experiment and simulation that the guided mode resonance wavelength $\lambda_{\mathrm{GMR}}$ does not coincide with the corresponding transmission maximum wavelength $\lambda_{\mathrm{OP}}$ in the orthogonal polarizer setup (which can equally be called a resonance wavelength), but differs by a few nanometres. Figure $4 \mathrm{~b}$ shows that the difference is $\Delta \lambda=8.3 \mathrm{~nm}$ for the investigated structure.

In the explanation presented above, we disregarded the phases of the TE and TM polarized light waves and considered $t_{\mathrm{TM}}$ and $t_{\mathrm{TE}}$ in $\mathcal{T}_{\mathrm{PCS}}$ to be real numbers. But the phase spectra of the TE and TM light in Fig. 5 show that during the TE resonance the TE phase changes rapidly, while the TM phase changes very slowly and smoothly. These phases will play the crucial part in deriving the origin of the deviating resonance wavelengths for the two different measurement setups. 
(1)
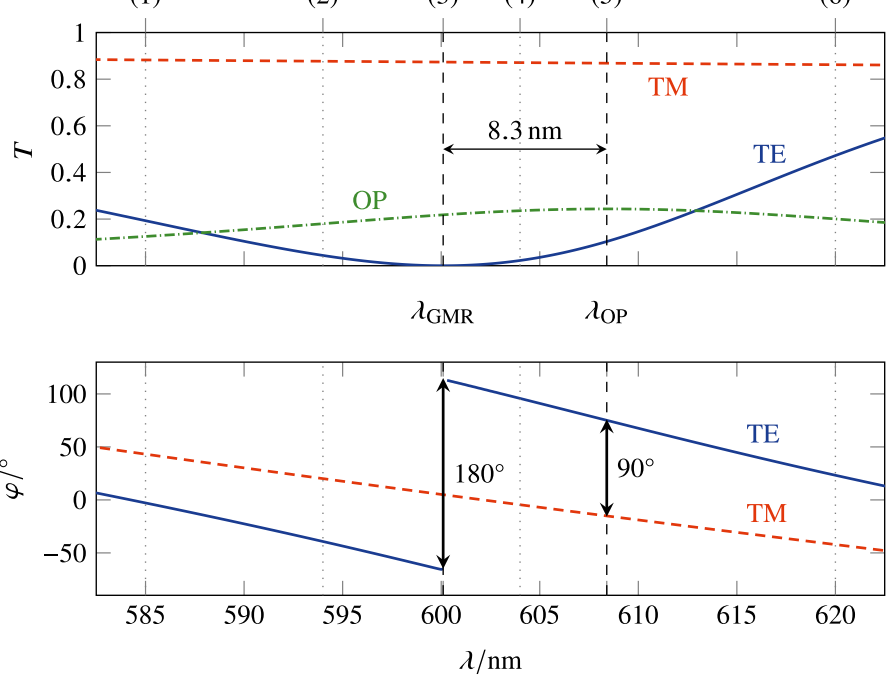

Fig. 5 FDTD results for the phase behaviour of the TE and TM fields at the TE resonance in detail. At $\lambda_{\mathrm{GMR}}$, the electric field's sign changes while passing the zero and the phase jumps by $180^{\circ}$. At $\lambda_{\mathrm{OP}}$, the TE and TM fields are out of phase by $90^{\circ}$. This leads to maximum transmission for the orthogonal polarizer setup, as shown with the polarization ellipses in Fig. 6. The numbers (1)-(6) refer to the six subplots in Fig. 6

Near the resonance, the large phase difference between TE and TM waves leads to elliptically polarized light behind the photonic crystal slab instead of linearly polarized light and the polarization ellipse rotates during the transition of the resonance, as shown in Fig. 6, where the polarization ellipse is depicted for different wavelengths around the TE resonance. To complement the qualitative discussion, numerical values for the Jones vectors $\vec{J}_{\text {PCS }}$ and $\vec{J}_{\text {out }}$ are given in Fig. 6 for all six ellipses. For example, the values for case (1) are calculated as follows: from Fig. 5 we see that $T_{\mathrm{TE}}=\left|t_{\mathrm{TE}}\right|^{2}=0.193, T_{\mathrm{TM}}=\left|t_{\mathrm{TM}}\right|^{2}=0.884$, and the phase difference is $\Delta \varphi=-46^{\circ}$. From that, we calculate

$$
\mathcal{T}_{\mathrm{PCS}}=\left(\begin{array}{cc}
t_{\mathrm{TM}} & 0 \\
0 & t_{\mathrm{TE}}
\end{array}\right)=\left(\begin{array}{cc}
0.940 & 0 \\
0 & 0.440 \mathrm{e}^{-\mathrm{i} 46^{\circ}}
\end{array}\right) \quad \text { and } \quad \vec{J}_{\mathrm{PCS}}=\mathcal{T}_{\mathrm{PCS}} \cdot \vec{J}_{\mathrm{in}}=\left(\begin{array}{c}
0.664 \\
0.311 \mathrm{e}^{-\mathrm{i} 46^{\circ}}
\end{array}\right) \text {. }
$$

Because only the phase difference between TE and TM is important, all calculated Jones vectors are normalized such that the first component is real. $\vec{J}_{\text {out }}=\mathcal{T}_{\text {Pol } 2} \cdot \vec{J}_{\text {PCS }}$ is presented such that the numerator is equal to the vector's modulus.

We partition this change of the ellipse's orientation into the following stages (the numbers in parentheses refer to Fig. 6):

- Relatively far from resonance, the major axis is oriented towards the first polarizer and only little light can pass the second polarizer (1).

- Approaching $\lambda_{\mathrm{GMR}}(2)$, the TE component vanishes and the ellipse reduces to a line (3), oriented at $45^{\circ}$ to both polarizers, such that half of the TM light can pass the second polarizer. Without considering the phases, we would expect the transmission maximum for the orthogonal polarizer setup here . 

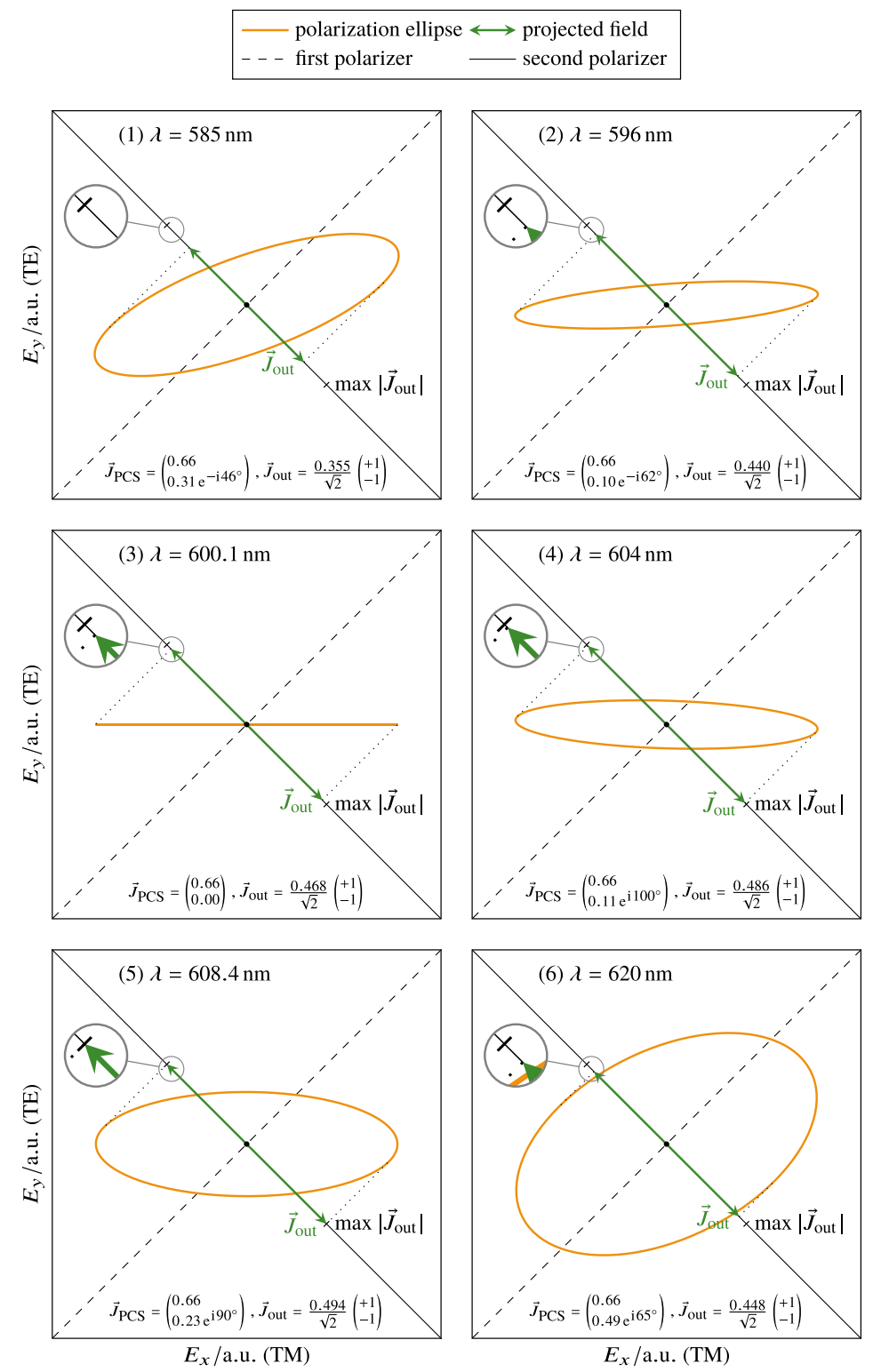

Fig. 6 Polarization ellipses for $\vec{J}_{\mathrm{PCS}}$, i.e. the light directly after the photonic crystal slab, for the different stages of the TE resonance. The ellipses have been calculated from the FDTD results. The numerical values for $\vec{J}_{\text {PCS }}$ and $\vec{J}_{\text {out }}=\mathcal{T}_{\text {Pol } 2} \cdot \vec{J}_{\text {PCS }}$ are shown at the bottom of each figure (the phases are always normalized such that $J_{1} \in \mathbb{R}$ ). The numbers (1)-(6) refer to the wavelengths indicated in Fig. 5 and to the description in Sect. 4. The orientation of the first and second polarizer are indicated with the dashed and solid diagonal lines. Only that part of the elliptically polarized light that is aligned to the second polarizer can pass it. Therefore, the projection of the ellipse onto the second polarizer is shown with dotted lines and the green arrows. These green arrows are the visual representation of $\vec{J}_{\text {out }}=\mathcal{T}_{\text {Pol } 2} \cdot \mathcal{T}_{\text {PCS }} \cdot \vec{J}_{\text {in }}$. The maximum projected field strength, reached at $\lambda_{\mathrm{OP}}$, is indicated with a tick on the second polarizer in all figures. Note that the green arrows only reach this tick for $\lambda=608.4 \mathrm{~nm}$, which corresponds to $\left|\vec{J}_{\text {out }}\right|=0.494 \approx 1 / 2$, and are shorter for every other wavelength, which can be seen in the magnification 


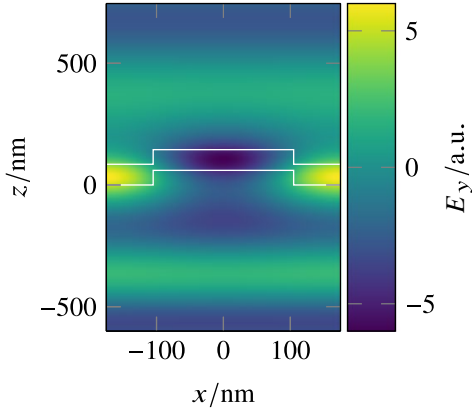

(a)

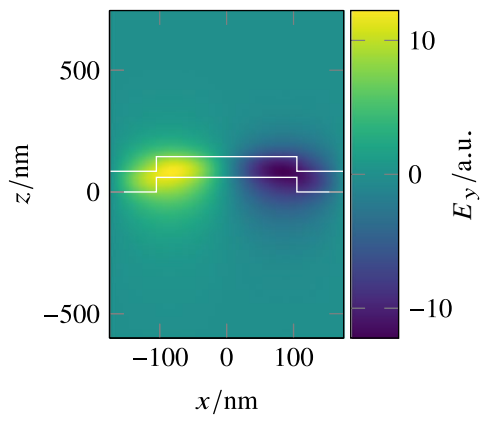

(b)

Fig. 7 Eigenmode analysis of the photonic crystal using the finite element method (COMSOL Multiphysics 2019). For the Bloch vector $k_{\mathrm{B}}$, two eigenmodes with eigenfrequencies $\left.\omega_{1}=3.0943+0.12529 \mathrm{i}\right) \times 10^{15} \mathrm{~s}^{-1}$ and $\omega_{2}=3.1466 \times 10^{15} \mathrm{~s}^{-1}$ exist, corresponding to $\lambda_{1}=608.7 \mathrm{~nm}$ and $\lambda_{2}=598.6 \mathrm{~nm}$. The first eigenmode [field profile shown in (a)] is a quasi-guided Bloch mode that couples to the outside. The second eigenmode [field profile shown in (b)] is a true guided Bloch mode that is not visible in the simulated transmission spectra

- At $\lambda_{\mathrm{GMR}}$, where $E_{y}=0$, the TE fields experience $180^{\circ}$ phase jump. Because of this sign change after $\lambda_{\mathrm{GMR}}$, the ellipse starts to tilt towards the second polarizer's orientation (4). The transmitted light after the second polarizer increases.

- When the phase difference between TE and TM decreases to $90^{\circ}$, the ellipses' major axis is again oriented at $45^{\circ}$ to both polarizers, but this time the ellipses is wide open due to the non-vanishing TE component (5). Here we have maximum transmission. We call this wavelength 'orthogonal polarizer resonance wavelength' $\lambda_{\mathrm{OP}}$.

- Further apart from the resonance, the phase difference between TE and TM approaches $0^{\circ}$ and the ellipses rotates back towards the first polarize's orientation (6): the transmission through the second decreases again.

Now one question arises: Which resonance wavelength is the 'correct' one? The 'guided mode resonance wavelength' $\lambda_{\mathrm{GMR}}$, where the transmission is zero, or 'orthogonal polarizer resonance wavelength' $\lambda_{\mathrm{OP}}$, where the transmission maximum with the orthogonal polarizer setup occurs? To answer this question, we performed a Bloch eigenmode analysis of the investigated structure for the Bloch wavevector $k_{\mathrm{B}}=0$, corresponding to perpendicularly incident light. The results are shown in Fig. 7. The calculated quasi-guided mode's eigenfrequency corresponds closely to $\lambda_{\mathrm{OP}}\left(\lambda_{\mathrm{QGM}}=608.7 \mathrm{~nm}\right.$ versus $\left.\lambda_{\mathrm{OP}}=608.4 \mathrm{~nm}\right)$. Therefore, the orthogonal polarizer setup has to be used to determine the quasi-guided mode's properties exactly.

\section{Comparison to other explanations of the resonance shape}

The explanation presented above is descriptive, because it is directly based on the behaviour of the calculated fields and is therefore well suited for explaining the origin of the resonance shapes. But it does not provide a method to predict the resonance shapes, because it relies on the beforehand calculated full fields. For that, temporal coupled-mode theory can 
be used, which provides an abstract way of explaining and also predicting the resonance shape based on just a few input parameters.

\subsection{Temporal coupled-mode theory}

It is known that the resonances in transmission and reflection spectra are Fano resonances, created by the interplay of non-resonant background light with the light leaking from the resonator (Wood 1902; Fano 1941).

Temporal coupled-mode theory is able to accurately model Fano resonances of photonic crystal slabs (Fan et al. 2003; Suh et al. 2004). We will briefly discuss how the formation of the resonance peak in the orthogonal polarizer setup is explained in this framework. We follow the work of Fan et al. (2003) with slightly adapted notation.

The coupled-mode equations for one resonator with multiple ports are

$$
\begin{aligned}
\frac{\mathrm{d} a}{\mathrm{~d} t} & =\left(\mathrm{i} \omega_{0}-1 / \tau\right) a+\vec{\kappa}^{\top} \cdot \vec{\psi}_{\text {in }} \\
\vec{\psi}_{\text {out }} & =C \cdot \vec{\psi}_{\text {in }}+a \vec{\kappa},
\end{aligned}
$$

where $a$ is the amplitude of the resonant mode, $\omega_{0}$ is the resonance's frequency, $\tau$ its lifetime, $\vec{\psi}_{\text {in }}$ and $\vec{\psi}_{\text {out }}$ the vectors of the in- and outgoing waves' amplitudes at all the ports, $\vec{\kappa}$ the coupling vector for the in- and outcoupling and $C$ is the matrix describing the direct pathways between the ports.

For external excitation, the scattering matrix $S$ for the whole system is

$$
\vec{\psi}_{\text {out }}=S \cdot \vec{\psi}_{\text {in }}=\left(C+\frac{\vec{\kappa} \cdot \vec{\kappa}^{\top}}{\mathrm{i}\left(\omega-\omega_{0}\right)+1 / \tau}\right) \cdot \vec{\psi}_{\text {in }} .
$$

In our case, the ports and waves are defined as shown in Fig. 8a. Because the TE and TM mode are orthogonal, their scattering processes are independent and we can write down the corresponding matrices $S^{\mathrm{TE}}$ and $S^{\mathrm{TM}}$ individually with $\kappa^{\mathrm{TE}}, \omega_{0}^{\mathrm{TE}}, \tau^{\mathrm{TE}}$ and $\kappa^{\mathrm{TM}}, \omega_{0}^{\mathrm{TM}}, \tau^{\mathrm{TM}}$, respectively. The matrix $C$ is identical for both polarizations because it describes the scattering of the equivalent unstructured planar layer stack and is therefore identical for TE and $\mathrm{TM}$ at normal light incidence.

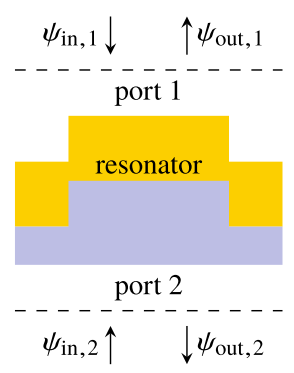

(a)

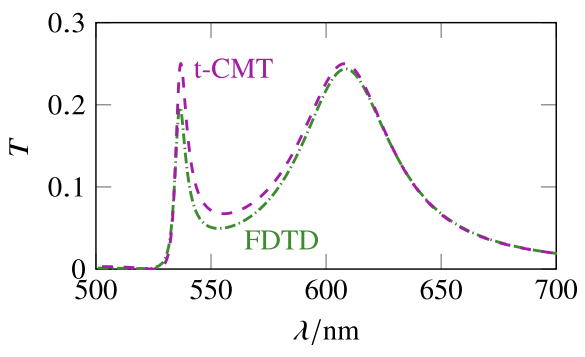

(b)

Fig. 8 a Definition of the ports and the waves in the temporal coupled-mode theory. The connection with the Jones vectors is $\vec{J}_{\text {in }}=\left(\psi_{\mathrm{in}, 1}^{\mathrm{TM}}, \psi_{\mathrm{in}, 1}^{\mathrm{TE}}\right)^{\mathrm{T}}$ and $\vec{J}_{\mathrm{PCS}}=\left(\psi_{\mathrm{out}, 2}^{\mathrm{TM}}, \psi_{\mathrm{out}, 2}^{\mathrm{TE}}\right)^{\mathrm{T}}$. b Comparison of the transmission spectrum calculated from temporal coupled-mode theory with the FDTD-simulated spectrum from Fig. 3. The t-CMT result has been calculated according to (Fan et al. 2003) with the following parameters: $\quad \omega_{0}^{\mathrm{TE}}=3.0943 \times 10^{15} \mathrm{~s}^{-1}, \quad \tau^{\mathrm{TE}}=7.9815 \times 10^{15} \mathrm{~s} \quad($ compare Fig. $7 \mathrm{a}), \quad \omega_{0}^{\mathrm{TM}}=3.5165 \times 10^{15} \mathrm{~s}^{-1}$, $\tau^{\mathrm{TM}}=5.4052 \times 10^{-14} \mathrm{~s}$ 
The transmission through the photonic crystals slab is described by the $S_{21}$ parameters. Therefore, its Jones matrix, written in the coupled-mode-theory framework, is

$$
\mathcal{T}_{\mathrm{PCS}}=\left(\begin{array}{cc}
S_{21}^{\mathrm{TM}} & 0 \\
0 & S_{21}^{\mathrm{TE}}
\end{array}\right)=\left(\begin{array}{cc}
C_{21}+\frac{\left(\vec{\kappa} \cdot \vec{\kappa}^{\mathrm{T}}\right)_{21}^{\mathrm{TM}}}{\mathrm{i}\left(\omega-\omega_{0}^{\mathrm{TM}}\right)+1 / \tau^{\mathrm{TM}}} & 0 \\
0 & C_{21}+\frac{\left(\vec{\kappa} \cdot \vec{\kappa}^{\top}\right)_{21}^{\mathrm{TE}}}{\mathrm{i}\left(\omega-\omega_{0}^{\mathrm{TE}}\right)+1 / \tau^{\mathrm{TE}}}
\end{array}\right) .
$$

The resulting polarisation state of the transmitted light is $\vec{J}_{\text {out }}=\mathcal{T}_{\mathrm{Pol} 2} \cdot \mathcal{T}_{\mathrm{PCS}} \cdot \vec{J}_{\text {in }}$, from which we calculate the transmission:

$$
T=\frac{\left|\vec{J}_{\text {out }}\right|^{2}}{\left|\vec{J}_{\text {in }}\right|^{2}}=\frac{1}{4}\left|+\frac{\left(\vec{\kappa} \cdot \vec{\kappa}^{\mathrm{T}}\right)_{21}^{\mathrm{TM}}}{\mathrm{i}\left(\omega-\omega_{0}^{\mathrm{TM}}\right)+1 / \tau^{\mathrm{TM}}}-\frac{\left(\vec{\kappa} \cdot \vec{\kappa}^{\mathrm{T}}\right)_{21}^{\mathrm{TE}}}{\mathrm{i}\left(\omega-\omega_{0}^{\mathrm{TE}}\right)+1 / \tau^{\mathrm{TE}}}\right|^{2} .
$$

Here we clearly see that for each polarization a Lorentzian with center frequency $\omega_{0}$ remains after the second polarizer because the two direct pathways $C$ cancel out. The two Lorentzians are also clearly visible in the graph of the above equation shown in Fig. 8b. This confirms that the 'orthogonal polarizer resonance wavelength' $\lambda_{\mathrm{OP}}$ is the correct wavelength that characterizes the resonantly interacting quasi-guided mode.

Another way of separating the resonant light from the background light can be performed in the time-domain. This is described in the next section.

\subsection{Time-domain apodization}

It has been demonstrated that in a time-domain simulation, for example with the FDTD method, the excitation of a photonic crystal slab with a broadband pulse leads to the following effect: The directly transmitted part of the light reaches the detector below the structure quickly, while another part excites the resonator and leaks slowly into the free space. This is shown in Fig. 9a. When the discrete Fourier transform (DFT) of the time-domain signal at the bottom detector is calculated, both parts of the light interfere and create the dip in the transmission spectrum at $\lambda_{\mathrm{GMR}}$. However, when the directly transmitted light is removed from the time-domain signal with a window function, which is also called apodization function, the interference vanishes and the light leaking from the resonator creates a Lorentzian-like peak in the spectrum (Fan and Joannopoulos 2002; Nazirizadeh 2010).

The example in Fig. $9 \mathrm{~b}$ shows that the resonance peak obtained by time-domain apodization is not equal to the resonance peak from the orthogonal polarizer setup. Especially the intensities differ. The peak positions differ slightly $(\Delta \lambda=0.7 \mathrm{~nm})$, despite manual finetuning of the apodization time (both peak position and intensity depend strongly on the settings for the apodization function). Nevertheless, the time-domain analysis provides an intuitive understanding of the resonance phenomenon.

\section{Summary}

We showed that the 'resonance wavelength' obtained with an orthogonal polarizer setup differs from the 'guided mode resonance wavelength' by a few nanometres. This has to be taken into account when determining parameters of the quasi-guided mode from a 


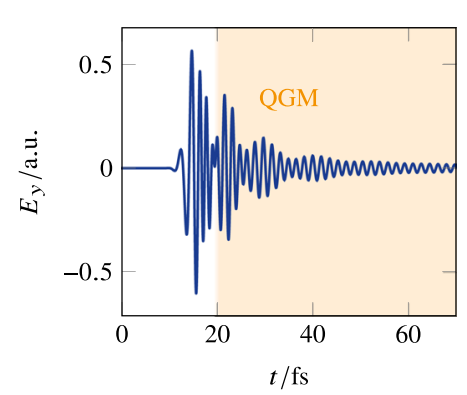

(a)

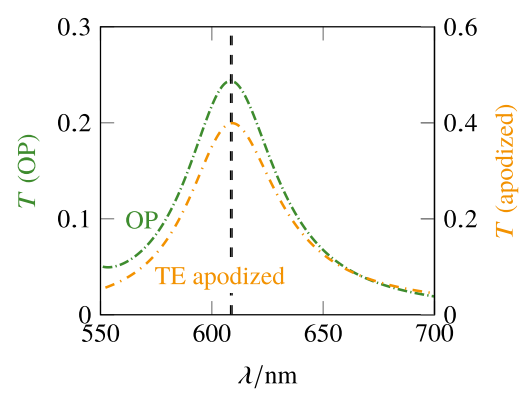

(b)

Fig. 9 a Time domain signal of the transmitted field. In the first 20 fs the direct pulse reaches the detector. After that, the leaking fields from the excited quasi-guided mode are detected. b TE Transmission spectrum obtained from the time signal shown in a after removing the first $20 \mathrm{fs}$ compared to the transmission for the orthogonal polarizer setup. Note that the apodization signal has a different vertical scale

transmission measurement or simulation. We presented a descriptive explanation of the orthogonal polarizer setup's working principle and for the observed effect of the wavelength difference. The explanation is directly based on the observed fields and approaches the phenomenon from another viewing angle compared to the more abstract temporal coupled-mode based derivation.

Both approaches explain the resonance shape for the orthogonal polarizer setup and the wavelength difference $\Delta \lambda$. Interestingly, the temporal coupled-mode theory explanation expresses that the TE and TM light from the direct pathways cancel and the light coupled into the resonator creates the observed resonance peak, thereby predicting the Lorentzian shape and quantitatively the peak intensity, while our explanation declares that the observed resonance peak is formed by the non-resonant polarization, because in resonance the direct and the resonant part of the light cancel. Yet, in the end both approaches describe the same result and are therefore equally valid.

Acknowledgements Open Access funding provided by Projekt DEAL. The authors acknowledge support by Interreg (Project Rollflex, 1_11.12.2014). We thank Torben Karrock for valuable discussions about the orthogonal polarizer setup.

Open Access This article is licensed under a Creative Commons Attribution 4.0 International License, which permits use, sharing, adaptation, distribution and reproduction in any medium or format, as long as you give appropriate credit to the original author(s) and the source, provide a link to the Creative Commons licence, and indicate if changes were made. The images or other third party material in this article are included in the article's Creative Commons licence, unless indicated otherwise in a credit line to the material. If material is not included in the article's Creative Commons licence and your intended use is not permitted by statutory regulation or exceeds the permitted use, you will need to obtain permission directly from the copyright holder. To view a copy of this licence, visit http://creativecommons.org/licenses/by/4.0/.

\section{References}

COMSOL Multiphysics. https://www.comsol.com/wave-optics-module (2019)

Fan, S., Joannopoulos, J.D.: Analysis of guided resonances in photonic crystal slabs. Phys. Rev. B 65(23), $235112(2002)$

Fan, S., Suh, W., Joannopoulos, J.D.: Temporal coupled-mode theory for the fano resonance in optical resonators. JOSA A 20(3), 569-572 (2003) 
Fano, U.: The theory of anomalous diffraction gratings and of quasi-stationary waves on metallic surfaces (Sommerfeld's waves). J. Opt. Soc. Am. 31(3), 213-222 (1941). https://doi.org/10.1364/ JOSA.31.000213

Hecht, E.: Optics, 4th edn. Pearson Education, Addison-Wesley, San Francisco (2002)

Hermannsson, P.G., Vannahme, C., Smith, C.L.C., Kristensen, A.: Absolute analytical prediction of photonic crystal guided mode resonance wavelengths. Appl. Phys. Lett. 105(7), 071103 (2014). https://doi. org/10.1063/1.4893664

Jahns, S., Bräu, M., Meyer, B.O., Karrock, T., Gutekunst, S.B., Blohm, L., Selhuber-Unkel, C., Buhmann, R., Nazirizadeh, Y., Gerken, M.: Handheld imaging photonic crystal biosensor for multiplexed, labelfree protein detection. Biomed. Opt. Express 6(10), 3724-3736 (2015)

Joannopoulos, J.D., Johnson, S.G., Winn, J.N., Meade, R.D.: Photonic Crystals: Molding the Flow of Light, 2nd edn. Princeton University Press, Princeton (2008)

Johnson, S.G., Fan, S., Villeneuve, P.R., Joannopoulos, J.D., Kolodziejski, L.A.: Guided modes in photonic crystal slabs. Phys. Rev. B 60, 5751-5758 (1999). https://doi.org/10.1103/PhysRevB.60.5751

Kilic, O., Fan, S., Solgaard, O.: Analysis of guided-resonance-based polarization beam splitting in photonic crystal slabs. JOSA A 25(11), 2680-2692 (2008)

Kwon, M.: A numerically stable analysis method for complex multilayer waveguides based on modified transfer-matrix equations. J Light Technol 27(20), 4407-4414 (2009). https://doi.org/10.1109/ JLT.2009.2024089

Lin, S.F., Chang, F.C., Chen, Z.H., Wang, C.M., Yang, T.H., Chen, W.Y., Chang, J.Y.: A polarization control system for intensity-resolved guided mode resonance sensors. Sensors 14(3), 5198-5206 (2014)

Lumerical Inc. Lumerical FDTD Solutions Release 2019a. Comsol Multiphysics Version 5.4 (2019). https ://www.lumerical.com/tcad-products/fdtd/. Accessed 10 Sep 2019

Nazirizadeh, Y.: Photonic Crystal Slabs for Low-Cost Biosensors. KIT Scientific Publishing, Karlsruhe (2010)

Nazirizadeh, Y., Müller, J.G., Geyer, U., Schelle, D., Kley, E.B., Tünnermann, A., Lemmer, U., Gerken, M.: Optical characterization of photonic crystal slabs using orthogonally oriented polarization filters. Opt. Express 16(10), 7153-7160 (2008). https://doi.org/10.1364/OE.16.007153

Nazirizadeh, Y., Bog, U., Sekula, S., Mappes, T., Lemmer, U., Gerken, M.: Low-cost label-free biosensors using photonic crystals embedded between crossed polarizers. Opt. Express 18(18), 19120-19128 (2010)

Pitruzzello, G., Krauss, T.F.: Photonic crystal resonances for sensing and imaging. J. Opt. 20(7), 073004 (2018). https://doi.org/10.1088/2040-8986/aac75b

Quaranta, G., Basset, G., Martin, O.J.F., Gallinet, B.: Recent advances in resonant waveguide gratings. Laser Photon. Rev. 12(9), 1800017 (2018). https://doi.org/10.1002/lpor.201800017

Rosenblatt, D., Sharon, A., Friesem, A.A.: Resonant grating waveguide structures. IEEE J. Quantum Electron. 33(11), 2038-2059 (1997). https://doi.org/10.1109/3.641320

Saleh, B.E., Teich, M.C.: Fundamentals of Photonics. Wiley, Hoboken (2019)

Suh, W., Wang, Z., Fan, S.: Temporal coupled-mode theory and the presence of non-orthogonal modes in lossless multimode cavities. IEEE J. Quantum Electron. 40(10), 1511-1518 (2004)

van Bladel, J.G.: Electromagnetic Fields, 2nd edn. Wiley, Hoboken (2007)

Wang, S.S., Magnusson, R.: Theory and applications of guided-mode resonance filters. Appl. Opt. 32(14), 2606-2613 (1993). https://doi.org/10.1364/AO.32.002606

Wood, R.W.: On a remarkable case of uneven distribution of light in a diffraction grating spectrum. Proc. Phys. Soc. 18(1), 269 (1902)

Publisher's Note Springer Nature remains neutral with regard to jurisdictional claims in published maps and institutional affiliations. 